\title{
High frequency energy transfer in semi-active suspension
}

\author{
C. Collette*, A. Preumont \\ University of Brussels, Department of Mechanical Engineering and Robotics, 50 av. F.D. Roosevelt, B-1050 Brussels, Belgium
}

\section{A R T I C L E I N F O}

Article history:

Received 20 April 2009

Received in revised form

23 March 2010

Accepted 27 May 2010

Handling Editor: A.V. Metrikine

Available online 18 June 2010

\begin{abstract}
A B S T R A C T
This paper investigates the effect of unintended high frequency excitations generated by the semi-active sky-hook control algorithm on the isolation properties of a car suspension. Using a quarter car model, an energy transfer from low to high frequency is established. An alternative algorithm is presented in this paper, in order to achieve smooth variations of the control force. Compared with the continuous semi-active sky-hook, the algorithm has been found to provide a similar damping of the body resonance, but also a better isolation at high frequency and global comfort.
\end{abstract}

(c) 2010 Elsevier Ltd. All rights reserved.

\section{Introduction}

A classical (passive) suspension is constituted of elements having constant stiffness and damping properties. The design of the suspension (i.e. the choice of the most suitable values of the stiffness and damping) is concerned with (i) the car body acceleration for ride comfort, (ii) the tyre deflection for road holding and (iii) the suspension travel (rattle space) which must remain within fixed limits. An ideal suspension would minimize these three quantities for any road and operating condition, which is not achievable for a suspension having constant stiffness and damping. Actually, there is a trade-off between ride comfort and road holding, or between the ride comfort and the suspension deflection [1,2].

A remedy to relax the trade-off is to use an active suspension [1,3]. In this case, the suspension can be either fully active, i.e. constituted of a wide band actuator capable of controlling the system in the full bandwidth, or passive on which is added such an actuator. Up to now, active suspensions are not widely used in the automotive industry because of their high external energy demand and complex actuation systems [4].

An alternative solution is to use a semi-active suspension [5,6]. In this case, the stiffness and damping properties can be changed in real time according to a chosen strategy. Requiring a minimum of effort, they are theoretically also able to reduce the car body resonance without degrading the road holding. This attractive solution leads unfortunately to some disturbances like jerk, chatter or rattling noise [7]. These problems have three origins: (i) a poor knowledge of the state variables of the system leading to some error of the command; (ii) an inability of the controlling device (servo valves) to follow the command, introducing a time delay; (iii) intrinsic to the semi-active strategy.

This paper focuses on the third origin. Actually, this case arises for instance when fast switches of the command generate high variations of the damper force, leading to an unpleasant rattle sound each time the valve is opened or closed $[8,9]$. The valve motion does not only cause sound problems but also the entire bodywork of the car starts to vibrate at a rather high frequency due to these abrupt control actions. The nonlinear energy transfer responsible for these excitations will be studied in details. Many strategies and technological solutions have been developed during the last two decades to avoid or alleviate these problems [4,9-14]. Some of them are briefly reviewed in this work. Two of them are studied in

\footnotetext{
* Corresponding author. Tel.: +3226504662; fax.:+3226504660.

E-mail address: ccollett@ulb.ac.be (C. Collette).
} 
details: filter the control input, and use a rubber bushing at both ends of the semi-active device to create a mechanical filter.

The paper is organized as follows. Section two compares the performances of a passive, active and semi-active suspension using a two dof model, which takes into account the mass of the wheel and the stiffness of the tyre. The effect of the unintended high frequency force excitations generated by the control algorithm on the suspension properties is pointed out. Section three reviews existing solutions to decrease the effect of these excitations.

In section four, an alternative algorithm is presented. The idea developed is to take advantage of dynamic properties of the car suspension to create a smooth command of the suspension. It will be shown that, while not optimal, this algorithm significantly improves the suspension performances at high frequency.

\section{2 dof suspension}

\subsection{Passive}

Fig. 1(a) shows the two dof quarter-car model with a passive suspension. The variables are: the vertical displacement of the sprung mass $m_{s}$ (representing one quarter of the car body mass), and the vertical displacement of the unsprung mass $m_{\mathrm{us}}$ (representing the wheel); $k_{t}$ is the stiffness of the tyre, $k$ and $c$ are the stiffness and damping coefficient of the suspension.

Using the set of state variables described in Fig. 1, the dynamics of the system read

$$
\begin{gathered}
m_{s} \dot{x}_{2}=-k x_{1}+c\left(x_{4}-x_{2}\right) \\
m_{\mathrm{us}} \dot{x}_{4}=-k_{t} x_{3}+k x_{1}+c\left(x_{2}-x_{4}\right) \\
\dot{x}_{1}=x_{2}-x_{4} \\
\dot{x}_{3}=x_{4}-v
\end{gathered}
$$

where $\dot{x}_{2}$ represents the ride comfort, $x_{3}$ the road holding, $x_{1}$ the rattle space and $v$ the input ground velocity, approximated by a white noise. The following numerical values have been used in this study: $m_{s}=240 \mathrm{Kg} ; m_{\mathrm{us}}=36 \mathrm{Kg} ; k_{t}=160000 \mathrm{~N} / \mathrm{m}$; $k=16000 \mathrm{~N} / \mathrm{m} ; c=1000 \mathrm{Ns} / \mathrm{m}$. A convenient quantity to compare the different designs is the RMS value of the relevant quantities; the RMS value of a variable $q$ is defined as

$$
\sigma_{q}=\left\{\int_{0}^{\infty} \Phi_{q}(\omega) \mathrm{d} \omega\right\}^{1 / 2}
$$

where $\omega$ is the frequency and $\Phi_{q}(\omega)$ the power spectral density of $q$. Fig. 2(a) shows $\sigma_{\ddot{x}_{s}}$ as a function of $\sigma_{x_{3}}$ when the damping coefficient of the suspension varies from $c=200$ to $5000 \mathrm{Ns} / \mathrm{m}$; Fig. 2(b) shows $\sigma_{\ddot{x}_{s}}$ as a function of $\sigma_{x_{1}}$ when the damping coefficient of the suspension varies from $c=200$ to $5000 \mathrm{Ns} / \mathrm{m}$.

Low values of $\sigma_{x_{3}}$ and $\sigma_{x_{1}}$ are obtained by increasing the damping coefficient, at the cost of an increase of $\sigma_{\ddot{x}_{s}}$. In other words, there exist a trade-off between the comfort and the road holding, and between the comfort and the suspension deflection. A remedy to relax the trade-offs is the use of active suspension [1,3] discussed in the next section.

(a)

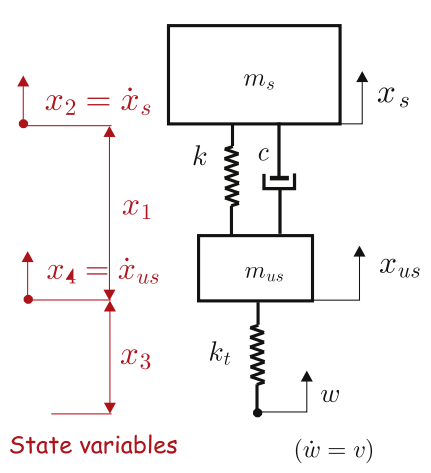

(b)

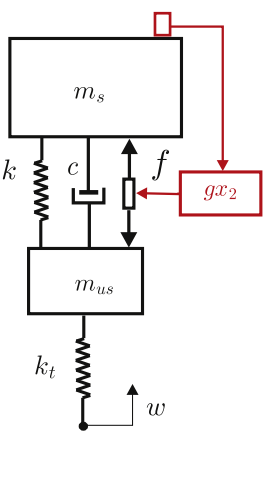

(c)

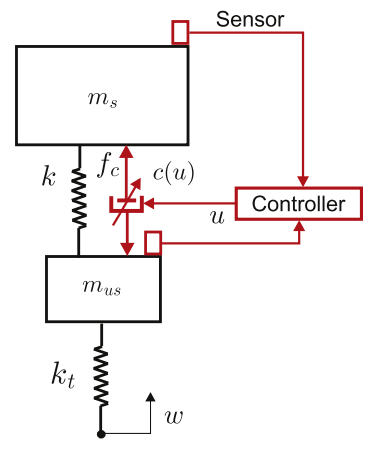

Fig. 1. Quarter-car model: (a) passive suspension; (b) active suspension; (c) semi-active suspension. 
(a)

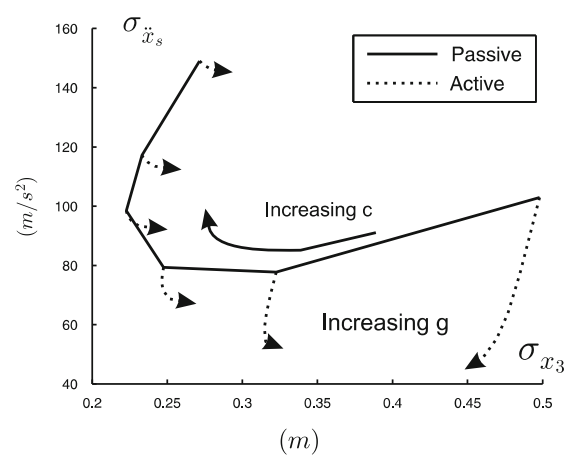

(b)

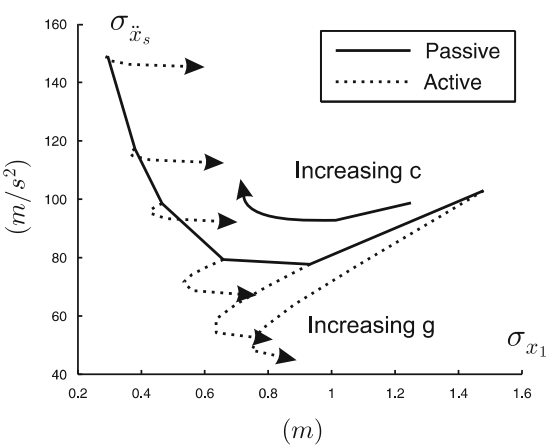

Fig. 2. (a) Trade-off curves between RMS value of the sprung mass acceleration $\sigma_{\ddot{x}_{s}}$ (representing the comfort) and RMS value of the tyre deflection $\sigma_{x_{3}}$ (representing the road holding). (b) Trade-off between RMS value of the sprung mass acceleration $\sigma_{\tilde{x}_{s}}$ and RMS value of the suspension deflection $\sigma_{x_{1}}$. Solid lines correspond to a passive suspension for which the value of $c$ increases from 200 to 5000 Ns/m; dashed lines correspond to an active suspension for which the value of the gain $g$ increases from 0 to $2000 \mathrm{Ns} / \mathrm{m}$.

\subsection{Active}

The active suspension can be either fully active, i.e. constituted of a wide band actuator capable of controlling the system in the full bandwidth, or a passive suspension supplemented with an ideal force actuator and controlled with a partial state feedback (e.g. sky-hook damper), as depicted in the Fig. 2(b). The dynamics of the system read

$$
\begin{gathered}
m_{s} \dot{x}_{2}=f-k x_{1}+c\left(x_{4}-x_{2}\right) \\
m_{\mathrm{us}} \dot{x}_{4}=-f-k_{t} x_{3}+k x_{1}+c\left(x_{2}-x_{4}\right) \\
\dot{x}_{1}=x_{2}-x_{4} \\
\dot{x}_{3}=x_{4}-v
\end{gathered}
$$

A classical control force is

$$
f_{c}=-g \dot{x}_{S}
$$

where $g$ is the gain. This law is called the sky-hook because it is identical to a viscous damper of constant $g$, connecting the sprung mass and a fixed point in space (the sky). Using this model, Fig. 2(a) shows, for each value of the damping coefficient considered, the evolution $\sigma_{\ddot{x}_{s}}$ as a function of $\sigma_{x_{3}}$ when the gain of the actuator increases from $g=0 \mathrm{Ns} / \mathrm{m}$ to $2000 \mathrm{Ns} / \mathrm{m}$. Similarly, Fig. 2(b) shows the evolution of $\sigma_{\ddot{x}_{s}}$ as a function of $\sigma_{x_{1}}$ when the gain of the actuator increases from $g=0$ to $2000 \mathrm{Ns} / \mathrm{m}$. One sees that, whatever the value of the damping coefficient, an increase of the gain always increases the comfort. However, very high values of the gain sometimes slightly degrade the suspension deflection or the road holding (Figs. 2(a) and (b)).

\subsection{Semi-active}

The semi-active suspension (Fig. 1(c)) is made of a spring, in parallel with a damper whose coefficient can vary in a fixed range. Sensors measure the state of the system in real time, and the control unit can change the value of the damping coefficient in a range between two extremal values $C_{\min }$ and $C_{\max }$, according to a defined strategy. As a nonlinear device, the response of such a shock absorber depends on the excitation amplitude and on its frequency content, and it has the capability to transfer energy from one frequency to another. Various classes of semi-active strategies are available in the literature [15-18]. The most often used is the semi-active sky-hook damper that tries to emulate the active sky-hook damper described in the previous section. Actually, the force in the damper is the product of its damping coefficient and the relative velocity of both ends:

$$
f_{c}=-c(u)\left(\dot{x}_{s}-\dot{x}_{\mathrm{us}}\right)
$$

where $c(u)$ is the damping coefficient, $\dot{x}_{s}$ the velocity of the sprung mass and $\dot{x}_{\mathrm{us}}$ the velocity of the wheel. Comparing Eqs. (3) and (2), the command that emulates the sky-hook damper is

$$
c(u)=\frac{g \dot{x}_{s}}{\dot{x}_{s}-\dot{x}_{\mathrm{us}}}(\geq 0)
$$


When $\dot{x}_{s}$ and $\dot{x}_{s}-\dot{x}_{\text {us }}$ have opposite sign, Eq. (4) leads to a negative value of $c(u)$. In this case, the damper force is fixed at its minimum value $C_{\min }$. Accounting that the maximum achievable coefficient is $C_{\max }$, the final control algorithm, often called SA1 in the literature, is

$$
c(u)=\max \left\{C_{\min }, \min \left[\frac{g \dot{x}_{s}}{\dot{x}_{s}-\dot{x}_{\mathrm{us}}}, C_{\max }\right]\right\}
$$

Time domain simulations have been conducted with the same numerical data as the passive suspension. The shock absorber constant is supposed to vary between $C_{\min }=100 \mathrm{Ns} / \mathrm{m}$ and $C_{\max }=2000 \mathrm{Ns} / \mathrm{m}$. The road velocity $v$ is assumed to be a white noise. Fig. 3 shows various time histories of the quarter-car response, respectively, the tyre force $k_{t} x_{3}$, the body velocity $\dot{x}_{s}=x_{2}$, the relative velocity between the body and the wheel $\dot{x}_{1}=\dot{x}_{s}-\dot{x}_{\text {us }}$, the required control force $f=-g \dot{x}_{s}$ and the actual control force $f_{c}=-c(u)\left(\dot{x}_{s}-\dot{x}_{\mathrm{us}}\right)$, and the damper constant $c(u)$.

Fig. 3 shows that each switch of the command creates a sudden change in force and acceleration of the suspended mass. Any sudden change of the seat acceleration is perceived by the passenger as a jerk. Fig. 4(a) compares the transmissibility between the road velocity and the body acceleration, $T_{\ddot{x}_{s} v}$ of, respectively, the passive suspension $(c=200 \mathrm{Ns} / \mathrm{m})$, the sky-hook control $(g=2000 \mathrm{Ns} / \mathrm{m})$ and the semi-active SA1 with $C_{\min }=100 \mathrm{Ns} / \mathrm{m}$ and $C_{\max }=2000 \mathrm{Ns} / \mathrm{m}$. The transmissibility has been obtained from a time simulation of $200 \mathrm{~s}$, with a time step of $0.0005 \mathrm{~s}$ and a white noise road velocity.

The figure shows two resonances. The first one, at $\omega_{1}=7.78 \mathrm{rad} / \mathrm{s}$, corresponds to a motion in phase of the two masses. As it is very close to the resonance of the body mass on the stiffness of the suspension (at $\omega_{n}=\sqrt{k / m_{s}}=8.16 \mathrm{rad} / \mathrm{s}$ ), it is commonly referred as the body resonance, or the sprung resonance. The second one, at $\omega_{2}=69.96 \mathrm{rad} / \mathrm{s}$, corresponds to a motion out of phase of the two masses. As it is very close to the resonance of the wheel on the stiffness of the tyre (at $\omega_{t}=\sqrt{k_{t} / m_{\mathrm{us}}}=66.67 \mathrm{rad} / \mathrm{s}$ ), it is commonly referred as the wheel resonance, or the unsprung resonance. One sees that the semi-active control reduces the body resonance, and the transmissibility of the body acceleration is comparable to
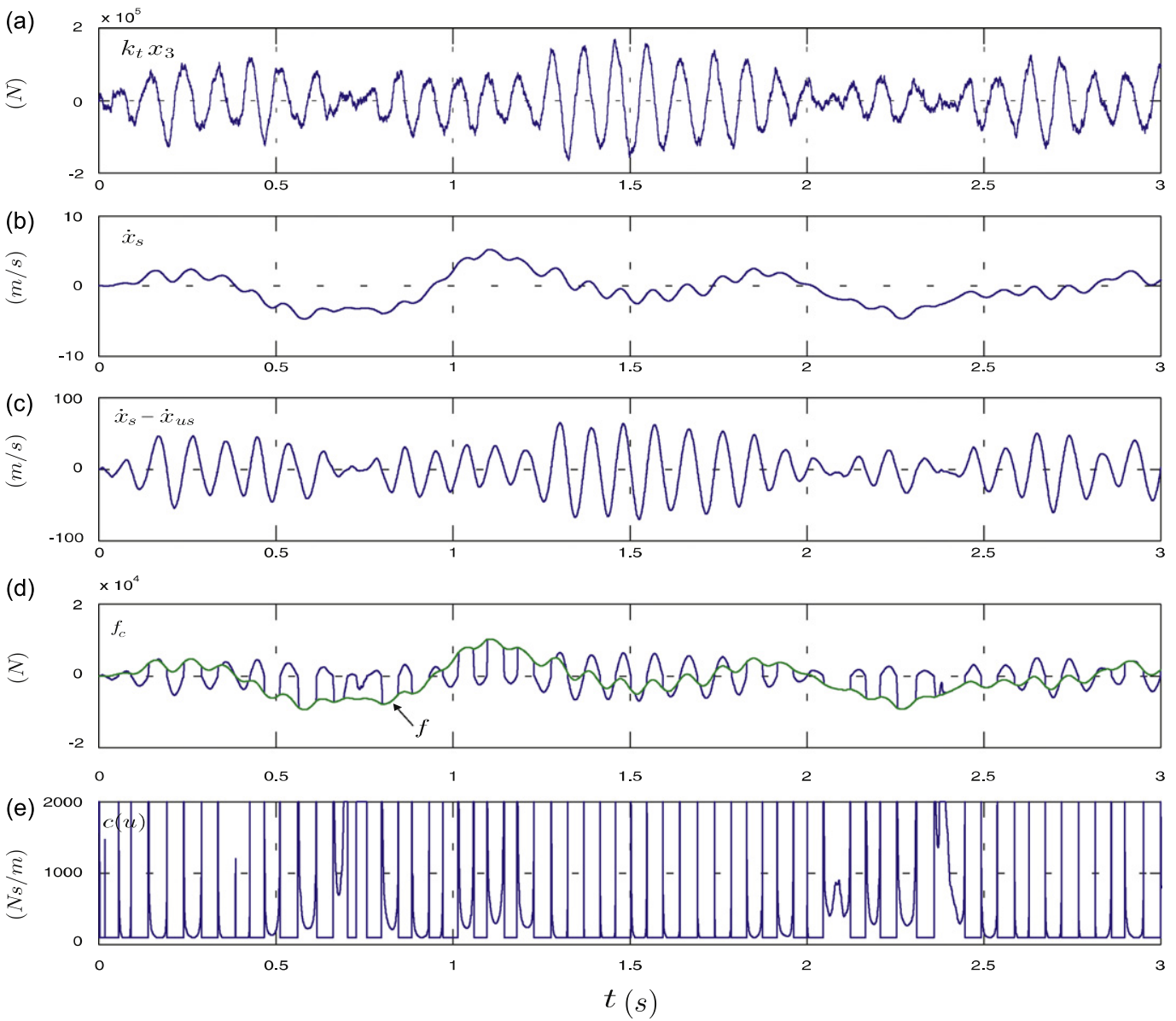

Fig. 3. Time history of a quarter-car model with a semi-active suspension using algorithm SA1. (a) Tyre force $k_{t} x_{3}$. (b) Body velocity $\dot{x}_{s}=x_{2}$. (c) Relative velocity between the body and the wheel $\dot{x}_{1}=\dot{x}_{s}-\dot{x}_{\text {us }}$. (d) Required force $f=-g \dot{x}_{s}$ and control force $f_{c}=-c(u)\left(\dot{x}_{s}-\dot{x}_{\text {us }}\right)$. (e) Damper constant $c(u)$. 

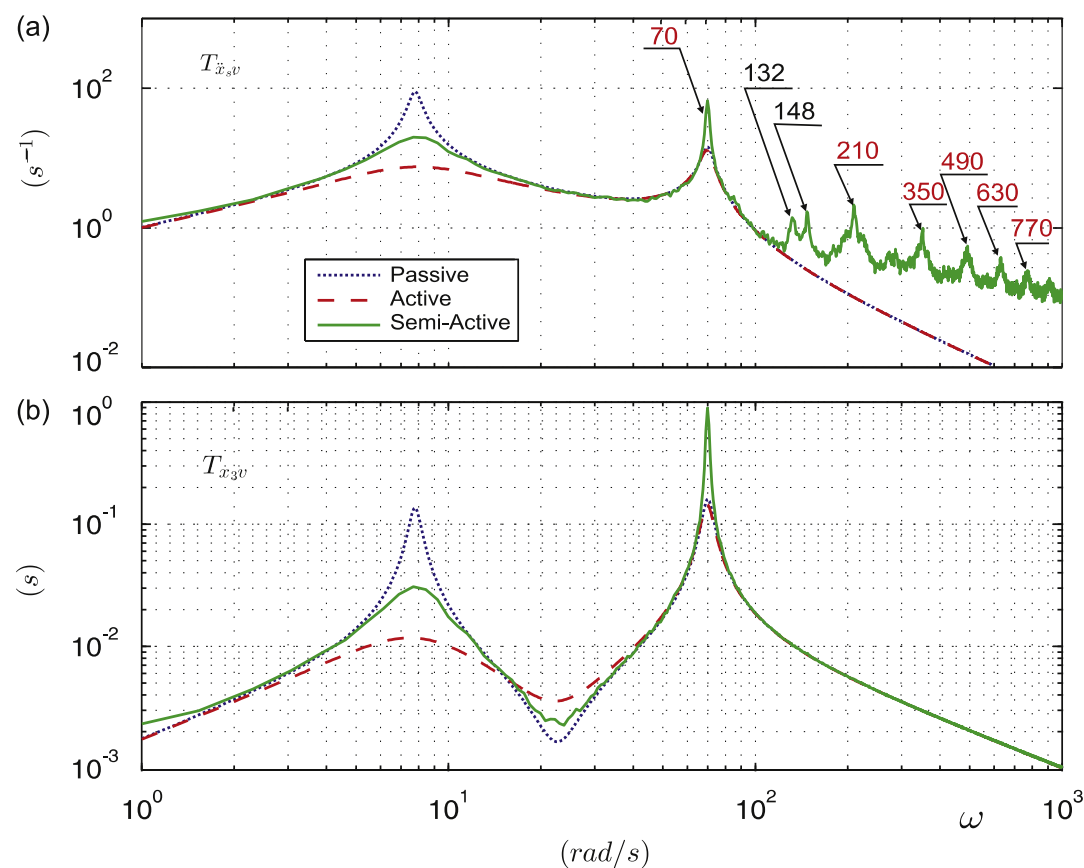

Fig. 4. Quarter car model with SA1 algorithm. (a) Transmissibility $T_{\ddot{x}_{s}}$ between the road velocity and the sprung mass acceleration: passive suspension ( $c=200 \mathrm{Ns} / \mathrm{m}$ ), active suspension $(g=2000 \mathrm{Ns} / \mathrm{m})$, semi-active sky-hook with $C_{\min }=100 \mathrm{Ns} / \mathrm{m}$ and $C_{\max }=2000 \mathrm{Ns} / \mathrm{m}$. (b) Transmissibility $T_{\chi_{3}} v$ between the road velocity and the tyre deflection.

that of the active control up to just before the wheel resonance. The wheel resonance is less damped than for the active and passive cases. Beyond that, the transmissibility rolls off much slower than in the active case, and one observes peaks at various harmonics of the wheel resonance, which are likely to excite flexible modes of the vehicle if nothing is done to attenuate them. Fig. 4(b) shows the transmissibility between the road velocity and the tyre deflection $T_{x_{3} v}$. No spurious high frequency components are observed and the road holding properties of the semi-active suspension seem to match closely to the active suspension, except the wheel resonance which is also more excited.

Due to the abrupt changes of the damping coefficient of the suspension when the sign of the relative velocity of the sprung and unsprung mass changes imposed by the SA1, harmonics of the second resonance of the suspension are visible on the sprung mass acceleration spectrum (Fig. 4(a)). The frequency of various peaks appearing in the transmissibility $T_{\ddot{x}_{s} v}$ is indicated. All components are at harmonics of the unsprung mode except for the second harmonic. The two peaks at 132.14 and $147.7 \mathrm{rad} / \mathrm{s}$ seem to result from the beat of the second harmonic of the wheel mode at $2 \omega_{2}=139.92 \mathrm{rad} / \mathrm{s}$ with the body mode at $\omega_{1}=7.78 \mathrm{rad} / \mathrm{s}$. The other peaks are odd harmonics of the second resonance, and the Fourier coefficients come directly from the decomposition of a square function $f(t)$ with a period $T$ into a sum of sine functions

$$
f(t)=\sum_{n=0}^{\infty} C_{n} \sin \left(\frac{2 \pi}{T}(2 n+1) t\right)
$$

where $C_{n}$ are constant coefficients. A first insight of the energy transfer from low to high frequencies is provided with the response of the semi-active suspension to a band-limited road velocity. Let us consider the road velocity shown in Fig. 5(a). It is a random noise, on which a low pass filter at $300 \mathrm{rad} / \mathrm{s}$ is applied.

Figs. 5(b) and (c) show respectively, the power spectral density of the sprung mass acceleration $\Phi_{\ddot{x}_{s} \ddot{x}_{s}}(\omega)$ and the power spectral density of the damper force $\Phi_{f_{c} f_{c}}(\omega)$. Above $300 \mathrm{rad} / \mathrm{s}$, no significant fall is visible on these curves. This means that the high frequency part of the spectrum does not result from a road excitation, but rather from the semi-active device itself.

Further evidence of the nonlinear energy transfer between low and high frequency can be obtained from the coherence function $\gamma_{v \dot{x}_{s}}^{2}(\omega)$ between the road velocity $v(t)$ and the sprung mass acceleration $\ddot{x}_{s}(t)$. It is defined by

$$
\gamma_{v \ddot{x}_{s}}^{2}(\omega)=\frac{\left|\Phi_{v \ddot{x}_{s}}(\omega)\right|^{2}}{\Phi_{v v}(\omega) \Phi_{\ddot{x}_{s} \ddot{x}_{s}}(\omega)}
$$

where $\Phi_{\ddot{\chi}_{s} v}(\omega)$ is the cross power spectral density, and shown in Fig. $6 . \gamma_{\dot{x}_{s} v}^{2}$ is equal to 1 for a perfect linear system without noise; it measures the causality of the two signals at every frequency. According to Fig. 6 , the coherence is very good up to the tyre mode, and falls rapidly to zero above $100 \mathrm{rad} / \mathrm{s}$, which indicates that at those frequencies, the energy content of the 


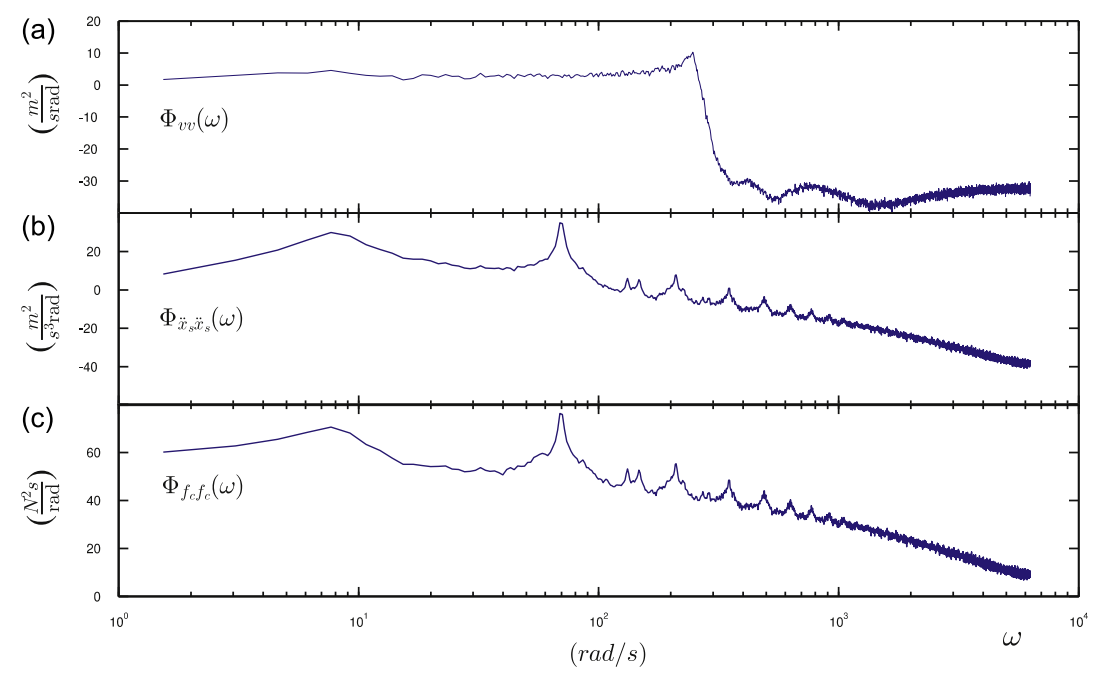

Fig. 5. Power spectral density of (a) the road velocity $\Phi_{v v}$, (b) the sprung mass acceleration $\Phi_{\ddot{x}_{s} \ddot{x}_{s}}$ and (c) the damping force $\Phi_{F_{c} F_{c}}$ for the semi-active suspension under a low frequency random excitation.

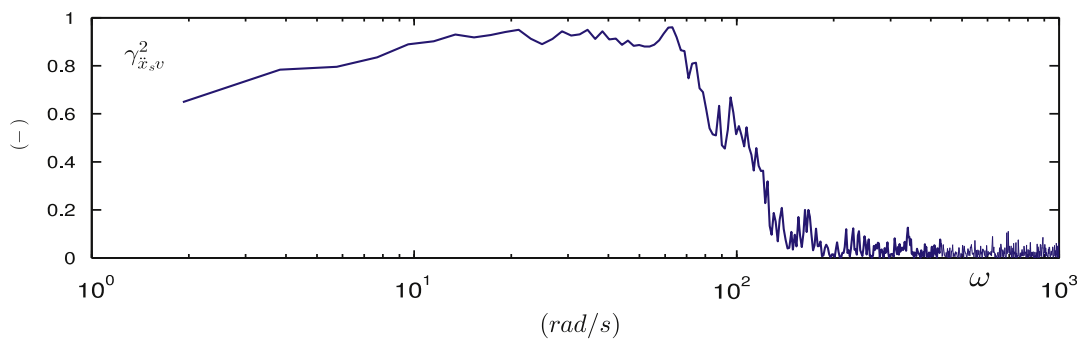

Fig. 6. Quarter car model with SA1 algorithm. Coherence function $\gamma_{\dot{x}_{s} v}^{2}$ between the road velocity $v(t)$ and the body acceleration $\ddot{x}_{s}(t)$.

body acceleration is not due to the road profile. The existing solutions to mitigate the jerk inherent to the algorithm SA1 are explained in the next section.

\section{Anti-jerk solutions}

\subsection{Modification of the SA1 algorithm}

Using the algorithm SA1, it has been pointed out in the previous section that the damping force is a discontinuous function of $\dot{x}_{s}$ and $\dot{x}_{s}-\dot{x}_{\text {us }}$ [5]. All of the discontinuities cause sudden variations that may lead to jerk. In order to reduce them, the control surface $f_{c}\left(\dot{x}_{s}, \dot{x}_{s}-\dot{x}_{\text {us }}\right)$ can be replaced by a continuous one, using a shaping function [19,7].

Another possible modification of the algorithm is to switch the valves only in those cases where the relative velocity between sprung and unsprung mass is zero [20]. In these instances, the suspension (spring + damper) force remains unchanged independent of the damper settings because no damper force can be generated [8].

\subsection{Change the damper parameters}

The range of variation of the damper constant has also an impact on the isolation properties of the suspension. High differences between $C_{\min }$ and $C_{\max }$ generate steps in the command and, as a consequence, high frequency components in the damper force. As the value of $C_{\max }$ increases, the saturation decreases. As a consequence, it increases slightly the damping of the first resonance, and increases the excitations at high frequencies. If the value of $C_{\text {min }}$ increases, the curvature of the damping force during the off period also increases. As a consequence, the switches between on and off show less sharp angles in the time history of the damper force. It decreases also the excitation of high frequencies and the semi-active damper becomes more and more passive. 

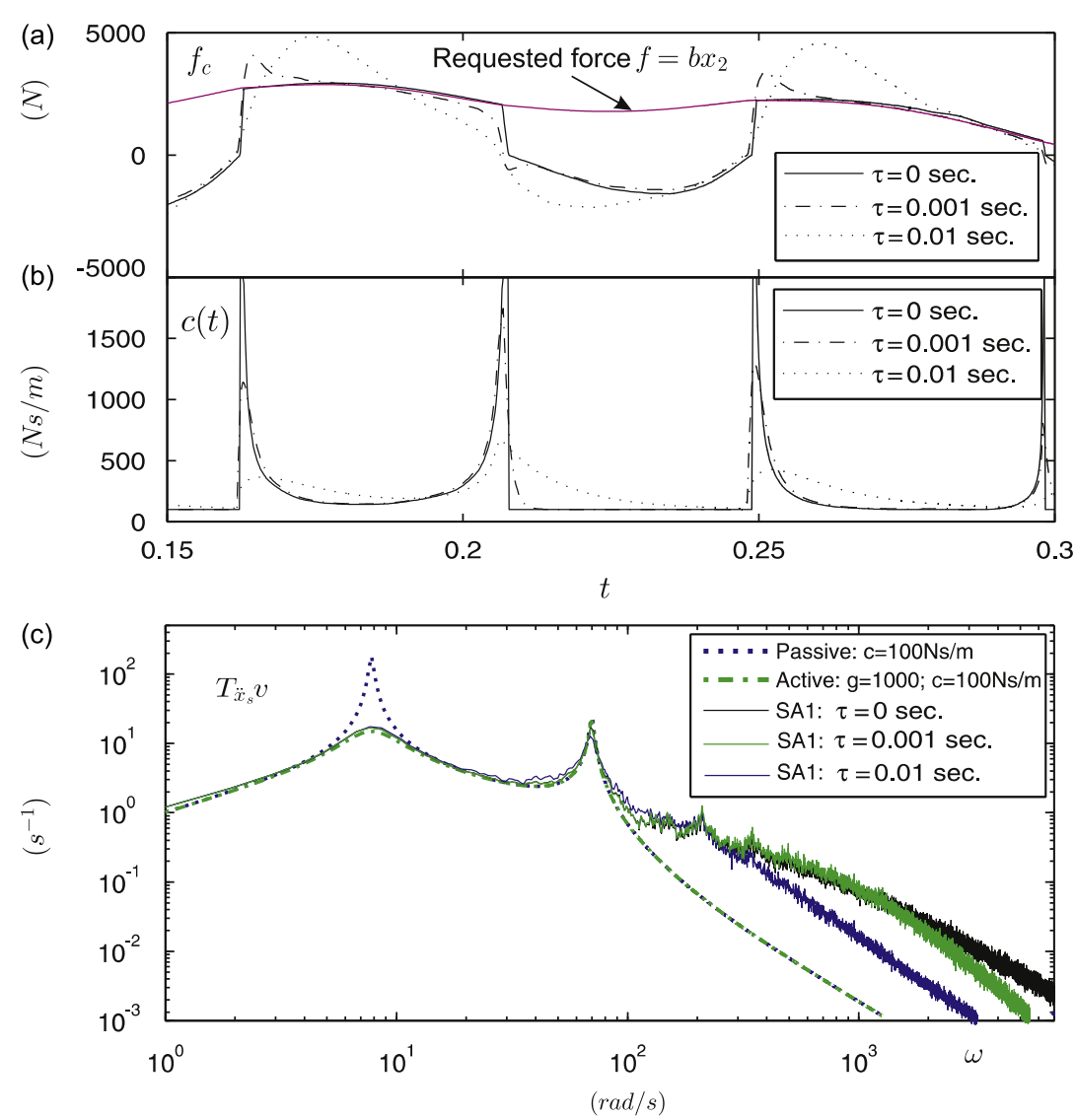

Fig. 7. Effect of the time delay on the suspension dynamics ( $g=2000 \mathrm{Ns} / \mathrm{m} ; C_{\min }=100 \mathrm{Ns} / \mathrm{m} ; C_{\max }=2000 \mathrm{Ns} / \mathrm{m}$ ): (a) detail of the damper force $f_{c}$ over a short period of time and corresponding value of the damping coefficient; (b) transmissibility $T_{\tilde{x}_{s} v}$ between the road velocity and the sprung mass acceleration. The transmissibilities are compared with the case of the passive suspension $(c=100 \mathrm{Ns} / \mathrm{m})$ and the active suspension $(c=100 \mathrm{Ns} / \mathrm{m}$ and $g=1000 \mathrm{Ns} / \mathrm{m})$.

\subsection{Filter the control input}

The introduction of a filter has an effect of smoothing the control force [4]. Fig. 7 shows the effect of a first-order filter $1 /(1+\tau s)$ for $\tau=0.01$ and $0.001 \mathrm{~s}$. As long as the bandwidth of the filter $(1 / \tau)$ is greater than the frequency of the second resonance $\left(\omega_{2}\right)$, the higher the value of $\tau$, the smoother the command, and the better the isolation of the sprung mass. The transmissibilities (Fig. 7(b)) are compared with the case of the passive suspension $(c=100 \mathrm{Ns} / \mathrm{m})$ and the active suspension $(c=100 \mathrm{Ns} / \mathrm{m}$ and $g=1000 \mathrm{Ns} / \mathrm{m}$ ). Whatever the constant of the filter is, the level of the transmissibility at high frequency remains higher for the semi-active suspension than for the active (and passive for $c=100 \mathrm{Ns} / \mathrm{m}$ ) suspension.

In practice, the time response of hydraulically powered digital servo valves lies in the range of $10 \mathrm{~ms}$ [8] and sometimes a little more [21]. As an alternative to an analog filter, the next section investigates if an elastic mount of the damper, using rubber bushing at both ends, could play a role of mechanical filter to smooth the control force.

\subsection{Effect of the rubber bushing compliance}

The bushing is modelled as a spring (Fig. 8, [10]). In this case, the dynamics of the system reads

$$
\begin{gathered}
m_{s} \dot{x}_{2}=-k x_{1}-k_{1} x_{5} \\
m_{\mathrm{us}} \dot{x}_{4}=k x_{1}+k_{1} x_{5}-k_{t} x_{3} \\
\dot{x}_{1}=x_{2}-x_{4} \\
\dot{x}_{3}=x_{4}-v \\
\dot{x}_{5}=x_{2}-x_{4}-\frac{k_{1}}{c} x_{5}
\end{gathered}
$$


and upon defining $\omega_{n}^{2}=k / m_{s}, \mu=m_{\mathrm{us}} / m_{\mathrm{s}}$ and $\omega_{t}^{2}=k_{t} / m_{\mathrm{us}}$ they are rewritten in matrix form

$$
\left(\begin{array}{l}
\dot{x}_{1} \\
\dot{x}_{2} \\
\dot{x}_{3} \\
\dot{x}_{4} \\
\dot{x}_{5}
\end{array}\right)=\left(\begin{array}{ccccc}
0 & 1 & 0 & -1 & 0 \\
-\omega_{n}^{2} & 0 & 0 & 0 & -\omega_{n}^{2} \kappa \\
0 & 0 & 0 & 1 & 0 \\
\omega_{n}^{2} / \mu & 0 & -\omega_{t}^{2} & 0 & \kappa \omega_{n}^{2} / \mu \\
0 & 1 & 0 & -1 & -k_{1} / c
\end{array}\right)+\left(\begin{array}{c}
0 \\
0 \\
-v \\
0 \\
0
\end{array}\right)
$$

The control algorithm is still defined by Eq. (5).

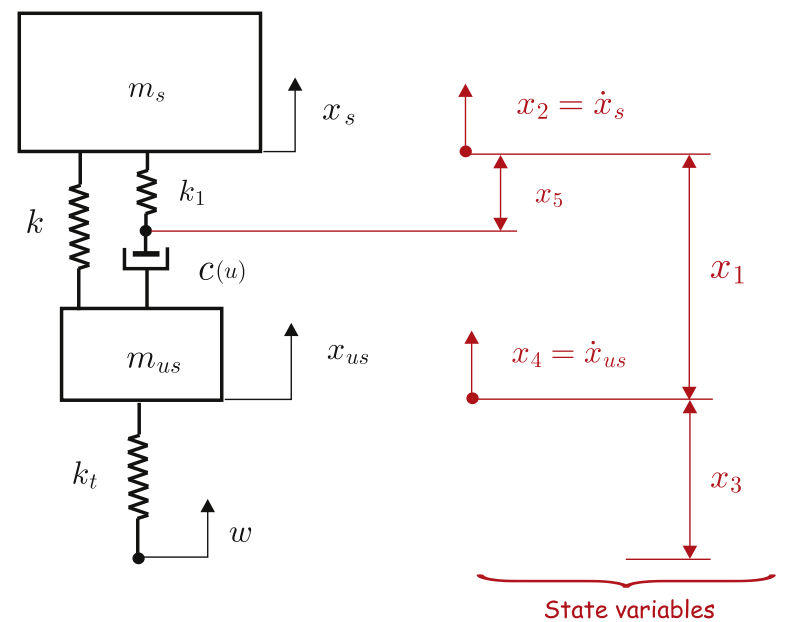

Fig. 8. State variables of the rubber bushing model.
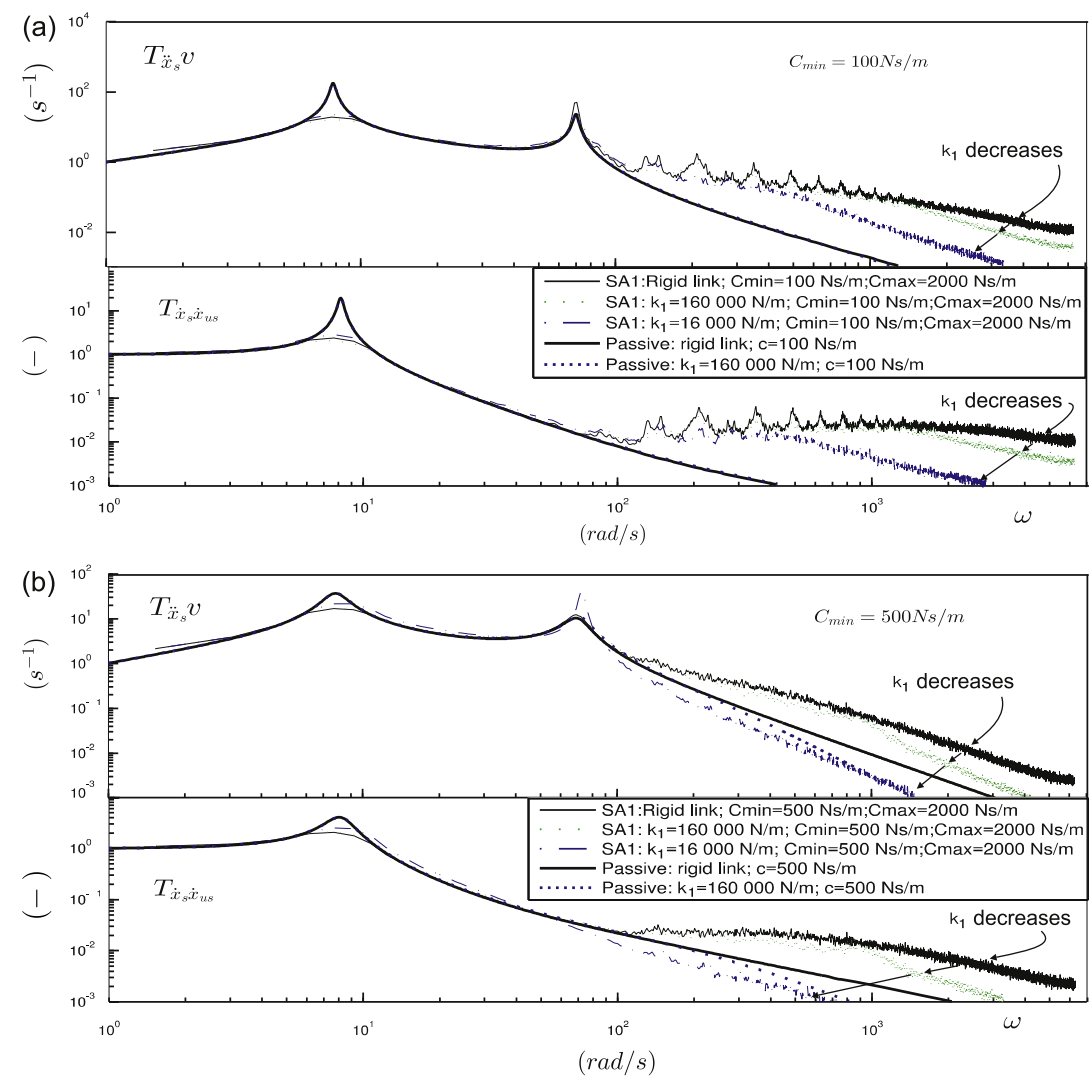

Fig. 9. Effect $k_{1}$ on the suspension transmissibility $T_{\dot{x}_{s} \dot{x}_{u s}}$ and $T_{\ddot{x}_{s} v}$ for (a) $C_{\min }=100 \mathrm{Ns} / \mathrm{m}$; (b) $C_{\min }=500 \mathrm{Ns} / \mathrm{m}\left(C_{\max }=2000 \mathrm{Ns} / \mathrm{m}\right)$. The lower the value of $k_{1}$, the better the isolation. 


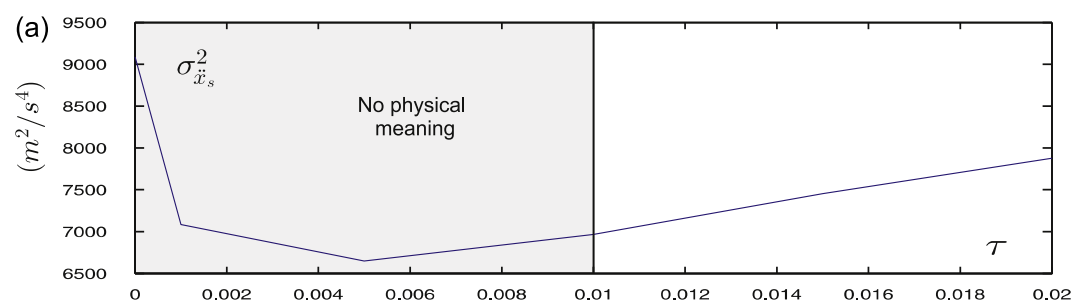

$(s)$

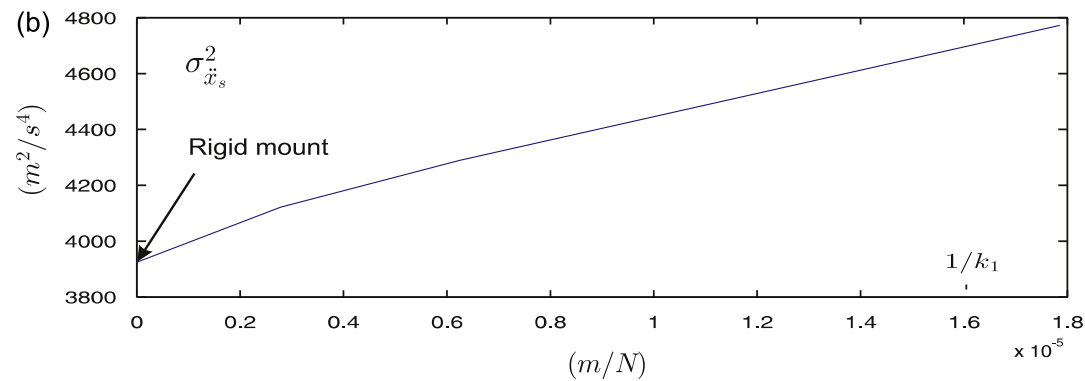

Fig. 10. (a) Mean square value of the sprung mass acceleration $\sigma^{2}\left(\ddot{x}_{s}\right)$ when the command passes through a first-order low pass filter as a function of the delay $\tau$ (the damper is rigidly mounted); (b) mean square value of the sprung mass acceleration $\sigma^{2}\left(\ddot{x}_{s}\right)$ as a function of the bushing compliance $\left(=1 / k_{1}\right)$.

Figs. 9(a) and (b) shows the effect $k_{1}$ on the suspension transmissibility $T_{\dot{x}_{s} \dot{x}_{\text {us }}}$ and the sprung mass acceleration transmissibility $T_{\ddot{x}_{s} v}$ for respectively, $C_{\min }=100$ and $500 \mathrm{Ns} / \mathrm{m}$.

Whatever the value of $C_{\max }$ is, a decrease of the bushing stiffness improves the isolation at high frequency. A low value of $C_{\min }$ generates high damper force variations each time the command switches and the peaks corresponding to harmonics of the second resonance are more sharp (Fig. 9, also section 3.2). In this case, the system is highly nonlinear and the effect of the bushing on the isolation is low. When the value of $C_{\min }$ is high (Fig. 9(b)), the suspension is more passive and the rubber bushing improves significantly the isolation, acting as a low-pass filter at high frequency. Thus, for a given value of $k_{1}$, the bushing provides a better isolation for high values of $C_{\min }$. This tends to say that the value of $k_{1}$ should be as low as possible. However, when the value $k_{1}$ becomes too low, the relaxation spring has an effect of increasing the level of the transmissibility $T_{\ddot{x}_{s} v}$ for frequencies below the second resonance. In order to choose the most suited value of $k_{1}$, Fig. 10 compares the effect of the filter and the bushing on the mean square value of the sprung mass acceleration $\sigma^{2}\left(\ddot{x}_{s}\right)$. Fig. 10(a) shows the mean square value of the sprung mass acceleration $\sigma^{2}\left(\ddot{x}_{s}\right)$ when the command passes through a first-order low pass filter as a function of the delay $\tau$. As the value of $\tau$ increases, the mean square value of the sprung mass acceleration decreases. For large values of $\tau$, it increases again. In this case, the optimal delay giving the lowest mean square of the sprung mass acceleration is $\tau=0.005 \mathrm{~s}$. Actually, such a criterion for the design of the filter is unrealistic because the optimal value is lower than the time response of the system (Section 3.3). In the case of a damper with elastic mounts, Fig. 10(b) shows that an increase of the bushing compliance $\left(=1 / k_{1}\right)$ increases the mean square of the sprung mass acceleration, for all value of the bush compliance.

In summary, both the filter and the bushing improve the quality of the high frequency isolation without deteriorating the behavior below the unsprung mass resonance, as long as the corner frequency of the filter is higher than the frequency of the second resonance of the suspension. However, none of these approaches seems to reduce the RMS sprung mass acceleration [22].

\section{Construction of the damper constant}

An example of time histories obtained using the semi-active sky-hook strategy is shown in Fig. 11. Each switch of the sign of the command (Fig. 11(d)) creates a sudden change in the damper force (solid line in Fig. 11(e)), perceived by the driver as jerk or rattling noise. In order to avoid these abrupt changes in the damper force between on and off states, an alternative approach would be to reinforce the value of the damper constant when the passivity condition is fulfilled (Eq. (4), dashed line in Fig. 11(d)), and reduce it when it is not. The resulting damper force would show the same variations as those obtained with the semi-active sky-hook, but smooth transitions (dashed line in Fig. 11(e)). The algorithm to construct such a signal is explained hereafter.

The sudden changes in the damper constant occur at each switch of the sign of

$$
y(t)=\dot{x}_{s}\left(\dot{x}_{s}-\dot{x}_{\mathrm{us}}\right)
$$



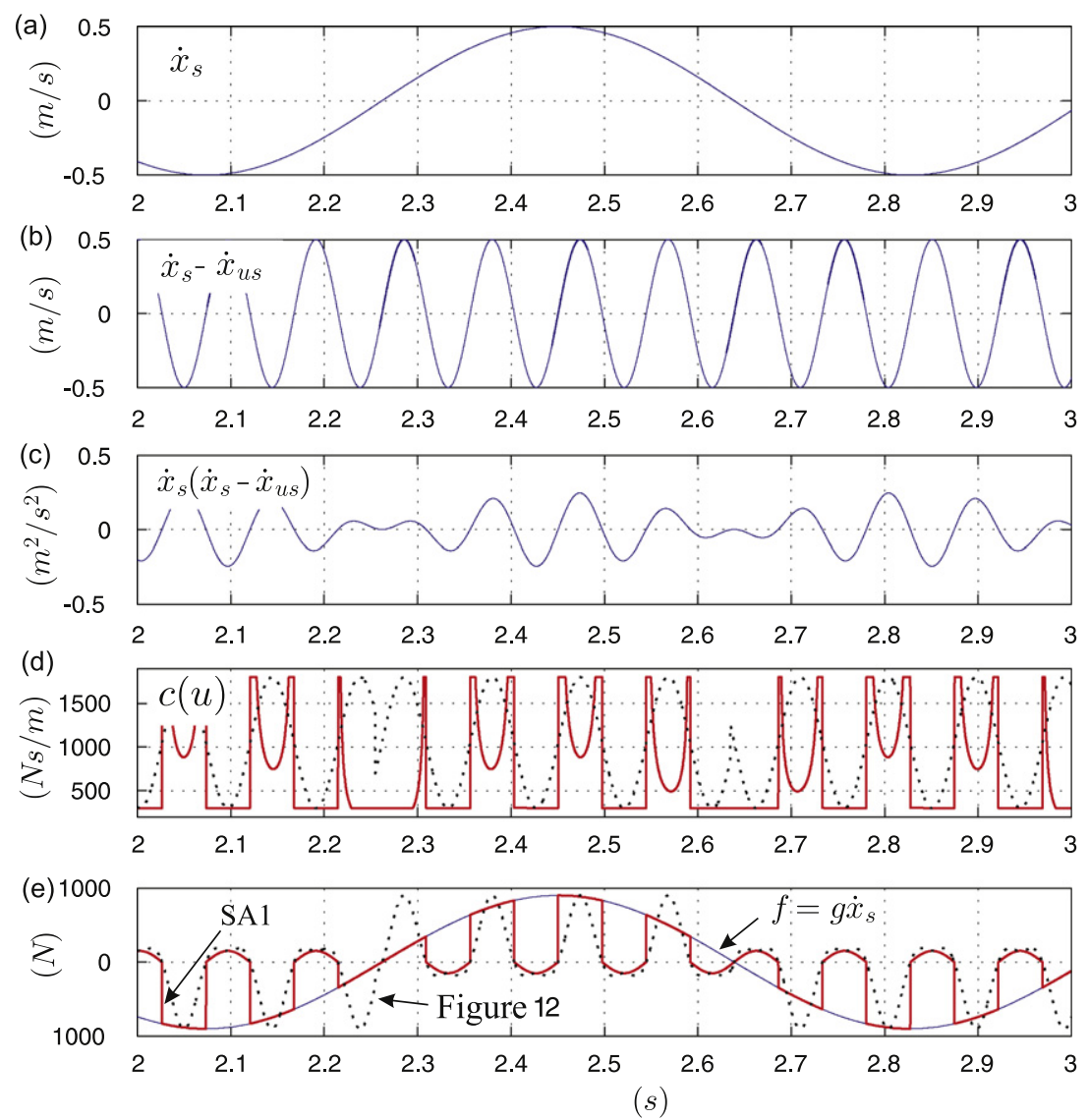

Fig. 11. (a) Sprung mass velocity $\dot{x}_{s}$; (b) suspension stroke $\dot{x}_{s}-\dot{x}_{\mathrm{us}}$; (c) $\dot{x}_{s}\left(\dot{x}_{s}-\dot{x}_{\mathrm{us}}\right.$ ); (d) damper constant $c(u)$ according to the SA1 algorithm (solid line) and according to Fig. 12 (dashed line); (e) comparison of the requested force $f=-g \dot{x}_{s}$ and two actual control forces: (i) when trying to emulate a low frequency signal $b \dot{x}_{S}$ with segments of $c(u)\left(\dot{x}_{s}-\dot{x}_{\text {us }}\right.$ ), i.e. SA1 strategy (solid line), (ii) smooth variations of the contact force (dashed line), obtained using the strategy described in Fig. 12.

Thus, the logical way to vary the value of the damper constant as mentioned above would be to impose to $c(t)$ the same variations around a constant value, i.e.

$$
c(t)=C_{0}+\Delta C y(t), \quad C_{\min }<c(t)<C_{\max }
$$

where $C_{0}$ and $\Delta C$ are constant quantities. The amplitudes of the variations are chosen by the gain $\Delta C$ : the higher the value of the gain, the more the sprung mass resonance is damped. As the value of $c(t)$ is bonded between $C_{\min }$ and $C_{\max }$, and as $y(t)$ is a zero mean process, the best performances of this algorithm are obtained for $C_{0}=\left(C_{\min }+C_{\max }\right) / 2$ and a high value of $\Delta C$. However, if the value of $\Delta C$ is too high, saturations will appear for high and low values of $c(t)$, because its variations are limited between $C_{\min }$ and $C_{\max }$. As a consequence, the strategy will look like the on-off semi-active sky-hook. On the other hand, if the value of $\Delta C$ is not high enough, this algorithm does not take fully advantage of the variability of the damper constant, because $c(t)$ does not systematically oscillate between the maximum and the minimum values when the passivity condition is fulfilled or not.

From these last considerations, we see that better performances will be achieved when $c(t)$ oscillates smoothly between $C_{\min }$ and $C_{\max }$, as depicted in Fig. 11(d). The strategy to construct such a signal is detailed hereafter.

Actually, $y(t)$ has a high frequency component $\dot{x}_{s}-\dot{x}_{\text {us }}$, varying around $\omega_{2}$, modulated by a low frequency signal $\dot{x}_{s}$, varying around $\omega_{1}$. As long as the carrier frequency $\omega_{2}$ is much higher than $\omega_{1}, y(t)$ is assumed to be a narrow band process. In this case, Eq. (9) can be rewritten in the form

$$
y(t)=A(t) \cos \left[\omega_{2} t+\theta(t)\right]
$$

where $A(t)$ and $\theta(t)$ are the amplitude and phase of the process. As we would like that $c(t)$ varies between $C_{\text {min }}$ and $C_{\text {max }}$ with a constant amplitude (i.e. $A(t)=1$ ), the expression of $c(t)$ becomes

$$
c(t)=C_{0}+\Delta C \cos \left[\omega_{2} t+\theta(t)\right]
$$


where $C_{0}=\left(C_{\min }+C_{\max }\right) / 2$ and $\Delta C=\left(C_{\max }-C_{\min }\right) / 2$. Assuming that $\omega_{2}$ has been determined beforehand (e.g. by modal testing), the only unknown quantity in Eq. (12) is the phase $\theta(t)$. It is extracted from $y(t)$ according to the following procedure (see Fig. 12).

Expanding Eq. (11), it can be written alternatively

$$
y(t)=\mathcal{C}(t) \cos \omega_{2} t-\mathcal{S}(t) \sin \omega_{2} t
$$

where $\mathcal{C}(t)=A(t) \cos \theta(t)$ and $\mathcal{S}(t)=A(t) \sin \theta(t)$ are called respectively, the cosine component and sine component of $y(t)$. Multiplying $y(t)$ by $2 \cos \omega_{2} t$, one gets

$$
W_{c}(t)=\mathcal{C}(t)+\left[\mathcal{C}(t) \cos \left(2 \omega_{2} t\right)-\mathcal{S}(t) \sin \left(2 \omega_{2} t\right)\right]
$$

and similarly, multiplying $y(t)$ by $2 \sin \omega_{2} t$ gives

$$
W_{s}(t)=-\mathcal{S}(t)+\left[\mathcal{C}(t) \sin \left(2 \omega_{2} t\right)+\mathcal{S}(t) \cos \left(2 \omega_{2} t\right)\right]
$$

Since $\mathcal{C}(t)$ and $\mathcal{S}(t)$ are slowly varying functions, the term inside the brackets of Eqs. (14) and (15) can be eliminated by low pass filtering to isolate respectively, the cosine $\mathcal{C}(t)$ and sine $\mathcal{S}(t)$ components. After filtering, these two components, varying at a frequency of $\omega_{1}$, are possibly affected by a constant phase lag $\phi\left(\omega_{1}\right)$ arising from the filter transmissibility.

Finally, the phase is constructed from the ratio between $\mathcal{S}(t)$ and $\mathcal{C}(t)$ by

$$
\theta(t)=\arctan \left\{\frac{\mathcal{S}(t)}{\mathcal{C}(t)}\right\}+\phi\left(\omega_{1}\right)
$$

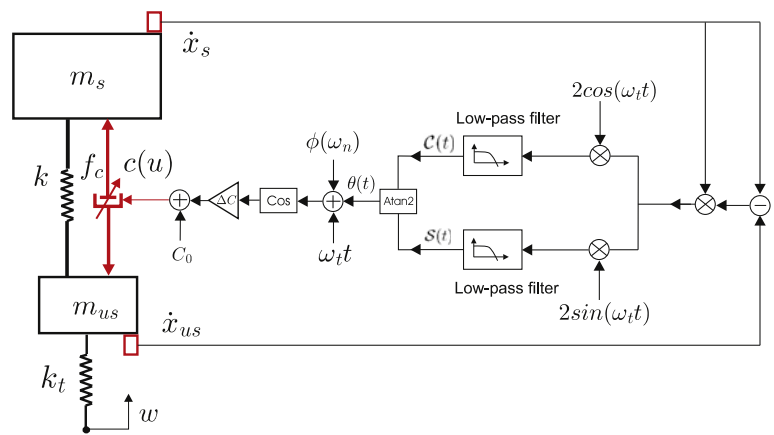

Fig. 12. Scheme of a controller imposing to the damper constant smooth transitions between $C_{\min }$ and $C_{\max }[23,24]$.
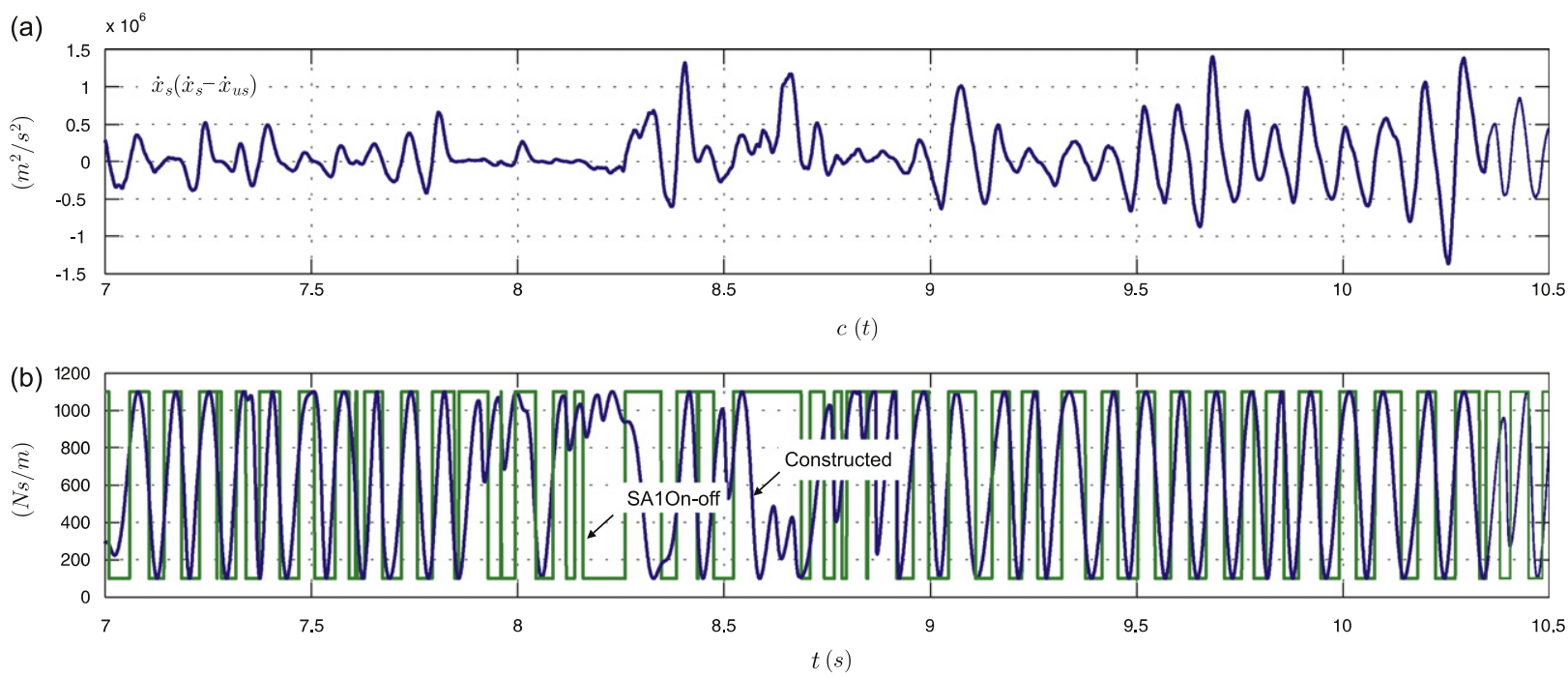

Fig. 13. Times histories of: (a) $\dot{x}_{s}\left(\dot{x}_{s}-\dot{x}_{\text {us }}\right)$; (b) damper constant constructed, compared with the on-off semi-active sky-hook strategy. For both algorithms $C_{\min }=100 \mathrm{Ns} / \mathrm{m}$ and $C_{\max }=1100 \mathrm{Ns} / \mathrm{m}$. 

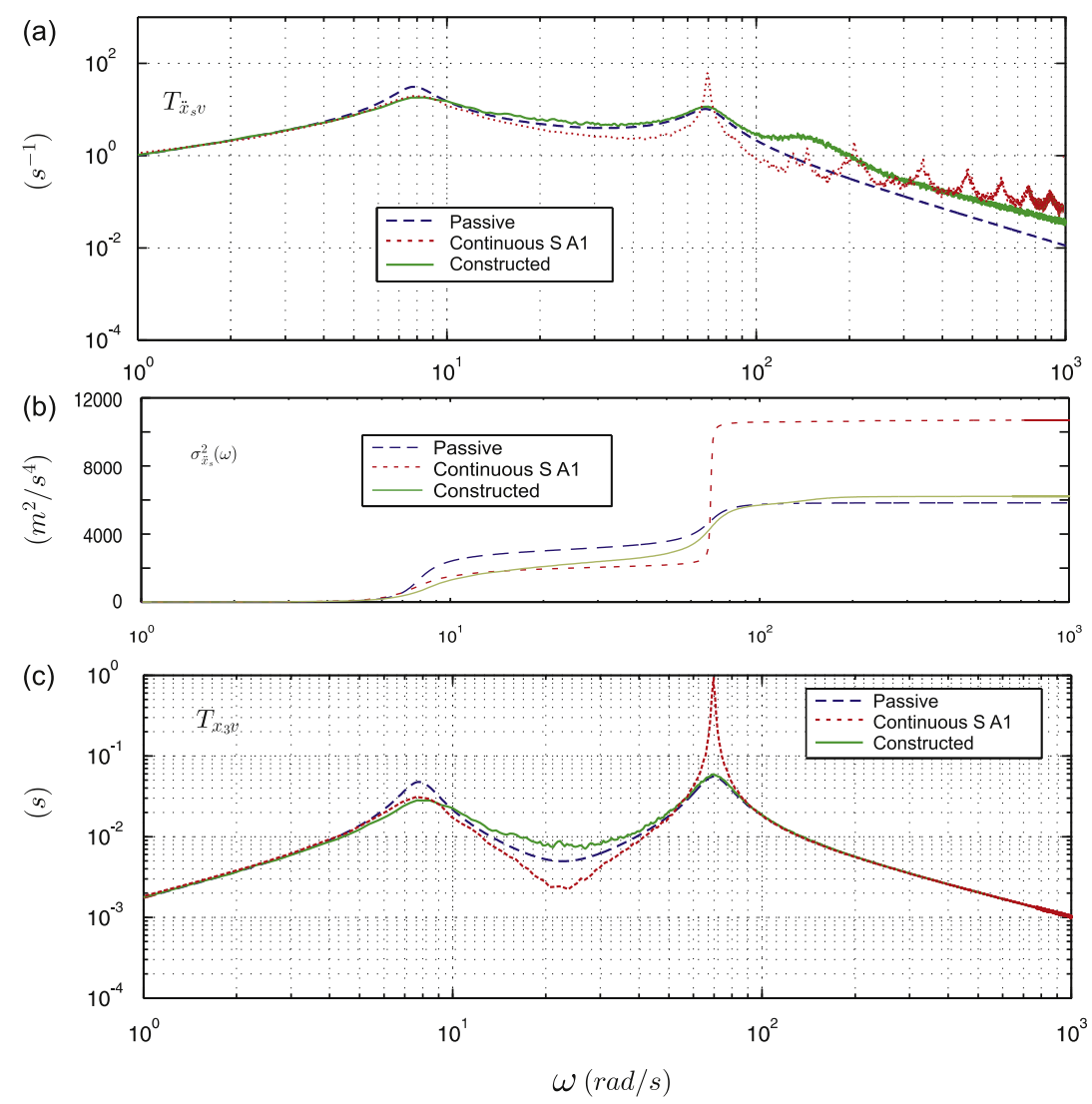

Fig. 14. Quarter car model with a semi-active suspension. (a) Transmissibility $T_{\ddot{\chi}_{s} v}$ between the road velocity and the sprung mass acceleration: passive suspension $(C=600 \mathrm{Ns} / \mathrm{m})$; Continuous semi-active sky-hook with $C_{\min }=100 \mathrm{Ns} / \mathrm{m}$ and $C_{\max }=1100 \mathrm{Ns} / \mathrm{m}$; Damper constant constructed, with $C_{\min }=100 \mathrm{Ns} / \mathrm{m}$ and $C_{\max }=1100 \mathrm{Ns} / \mathrm{m}$; (b) mean square value of the body acceleration $\sigma_{\tilde{x}_{s}}^{2}$; (c) transmissibility $T_{x_{3}} v$ between the road velocity and the tyre deflection.

Fig. 13 shows two time histories of the quarter car response, respectively, the product $\dot{x}_{s}\left(\dot{x}_{s}-\dot{x}_{\text {us }}\right)$ and the damper constant, constructed using the algorithm described above $\left(C_{\min }=100 \mathrm{Ns} / \mathrm{m}, C_{\max }=1100 \mathrm{Ns} / \mathrm{m}\right.$, Butterworth low pass filters of order 5).

For comparison, the history of the damper constant obtained using the on-off semi-active sky-hook is also shown in Fig. 13(b). When $\dot{x}_{s}\left(\dot{x}_{s}-\dot{x}_{\text {us }}\right)$ shows harmonic variations around $\omega_{2}$, the constructed damper constant varies smoothly from $C_{\min }$ to $C_{\max }$ (e.g. Fig. 13(b) between $t=9.5$ and $10.5 \mathrm{~s}$ ). However, because of the filtering, the algorithm is incapable of accommodating small variations at frequencies higher than $\omega_{2}$ (e.g. Fig. 13(b) between $t=7.8$ and $8.3 \mathrm{~s}$ ).

Fig. 14(a) compares the transmissibility between the road velocity and the body acceleration $T_{\ddot{x}_{s} \nu}$ of, respectively, the passive suspension $\left(C=C_{\min }+\Delta C / 2=600 \mathrm{Ns} / \mathrm{m}\right)$, the continuous semi-active sky-hook $\left(C_{\min }=100 \mathrm{Ns} / \mathrm{m}\right.$ and $\left.C_{\max }=1100 \mathrm{Ns} / \mathrm{m}\right)$ and the constructed dashpot constant $\left(C_{\min }=100 \mathrm{Ns} / \mathrm{m}\right.$ and $\left.C_{\max }=1100 \mathrm{Ns} / \mathrm{m}\right)$. The transmissibility has been obtained with a simulation of $200 \mathrm{~s}$, a time step of $0.0005 \mathrm{~s}$ and a white noise road velocity. Both semi-active strategies are able to reduce the body resonance at $\omega_{1}$, but the new strategy is additionally able to decrease the wheel resonance (like the passive suspension). On the other hand, the new algorithm degrades the ride comfort in the sensitive frequency range between 10 and 70 rad/s. At high frequency, none of them is able to provide the same isolation as the passive suspension. As shown in Fig. 14(b), they are also not able to decrease the mean square value of the sprung mass acceleration $\sigma_{\tilde{x}_{s}}^{2}$. Fig. 14(c) shows the transmissibility between road velocity and the tyre deflection $T_{x_{3} v}$. Regarding the damping of the two resonances, results are similar to Fig. 12(a); none of the semi-active strategies show spurious peaks at high frequency.

\section{Future work}

The results obtained from these numerical simulations are promising. However, the proposed algorithm obviously needs to be further improved and optimized. The main directions for future investigations are:

- Optimization of the algorithm and the parameters to improve the performances (e.g. add a band-stop filter to the command in the frequency range between 10 and $70 \mathrm{rad} / \mathrm{s}$ ). 
- Sensitivity analysis around the optimum parameters (e.g. impact of an error on $\omega_{2}$ ).

- Numerical validation under various operating conditions (e.g. shock or narrow band excitation).

- Experimental validation.

\section{Conclusion}

Through a simple model of a quarter-car, the isolation properties of a semi-active suspension has been studied using the SA1 sky-hook like algorithm. It has been shown that the isolation provided by the suspension is degraded by the nonlinearity of the semi-active algorithm, which tends to generate high frequency components at the harmonics of the unsprung mass resonance. These high frequency vibrations may potentially generate noise through the excitation of flexible modes of the vehicle. This energy transfer phenomenon may be alleviated to some extent by different means, like filtering the control signal or adding a resilient mounting to the semi-active damper.

In this paper, an alternative semi-active algorithm has been presented. By imposing smooth variations to the damper constant, it has been found that, for a similar reduction of the body resonance, the isolation at high frequency is improved compared to the continuous semi-active sky-hook. Additionally, the RMS value of the sprung mass acceleration is also reduced, mainly because of a better damping of the wheel resonance.

\section{Acknowledgements}

The authors gratefully acknowledge the reviewers for their useful comments, leading to substantial modifications of the manuscript and significant improvements of the overall quality of the paper.

\section{References}

[1] R. Chalasani, Ride performance potential of active suspension systems-part I: simplified analysis based on a quarter-car model, Transactions of the ASME 80 (1986) 187-204.

[2] D. Hrovat, Survey of advanced suspension developments and related optimal control applications, Automatica 33 (1997) $1781-1817$.

[3] D. Hrovat, Optimal active suspension structures for quarter-car vehicle models, Automatica 26 (1990) 845-860.

[4] D. Fischer, R. Isermann, Mechatronic semi-active and active vehicle suspensions, Control Engineering Practice 12 (2004) $1353-1367$.

[5] D. Karnopp, Design principles for vibration control systems using semiactive dampers, Transactions of the ASME, Journal of Dynamic Systems, Measurement and Control 112 (1990) 448-455.

[6] T. Gordon, R. Sharp, On improving the performance of automotive semi-active suspension systems through road preview, Journal of Sound and Vibration 217 (1998) 163-182.

[7] K. Liu, J. Liu, The damped dynamic vibration absorbers: revisited and new result, Journal of Sound and Vibration 284 (2005) 1181-1189.

[8] P. Venhovens, The development and implementation of adaptive semi-active suspension control, Vehicle System dynamics 23 (1994) 211-235.

[9] J. Lieh, Semi-active and active suspensions for vehicle ride control using velocity feedback, Journal of Vibration and Control 3 (1996) $201-212$.

[10] R. Henry, B. Murty, Quarter car variable force suspension system control, Patent No. US005276621A.

[11] M. Ahmadian, X. Song, S. Southward, No-jerk skyhook control methods for semiactive suspensions,, Transaction of the ASME, Journal of Vibration and Acoustics 126 (2004) 580-584.

[12] M. Ahmadian, Transient dynamics of semiactive suspensions with hybrid control, Journal of Intelligent Material Systems and Structures 17 (2006) 145-153.

[13] L. Miller, C. Nobles, Methods for eliminating jerk and noise in semi-active suspensions, SAE paper, 1990, pp. 943-951.

[14] S. Stamatov, M. Krishnan, S. Yost, Low jerk predictive force modulation for semi-active suspension control, in: SAE World Congress, 2008.

[15] F. Oueslati, S. Sankar, A class of semi-active suspension schemes for vehicle vibration control, Journal of Sound and Vibration 172 (1994) 391-411.

[16] T. Gordon, Nonlinear optimal control of a semi-active vehicle suspension system, Chaos, Solitons and Fractals 5 (1995) $1603-1617$.

[17] S. Ohsaku, T. Nakayama, I. Kamimura, Y. Motozono, Nonlinear $h_{\infty}$ control for semiactive suspension, JSAE Review 20 (1999) 447-452.

[18] Y. Liu, T. Water, M. Brennan, A comparison of semi-active damping control strategies for vibration isolation of harmonic disturbances, Journal of Sound and Vibration 280 (2005) 21-39.

[19] M. Ahmadian, R. Brian, X. Song, S. Southward, No-jerk semi-active sky-hook control method and apparatus, Patent No. US006115658A.

[20] R. Hanson, T. Radcliffe, Output filter and method for On/Off semiactive suspension control, U.S. Patent No. 5.024.460.

[21] N. Rensurg, J. Steyn, P. Els, Ime delay in a semi-active damper: modeling the bypass valve, Journal of Terramechanics 39 (2002) 35-45.

[22] C. Collette, A. Preumont, Energy transfer in semi-active suspension, in: International Conference on Noise and Vibration Engineering, ISMA2008, Leuven, 2008.

[23] S. Rice, Mathematical analysis of random noise, Bell System Technical Journal 23 (1944) 282.

[24] A. Preumont, Random Vibration and Spectral Analysis, Kluwer Academic Publishers, 1994. 